\section{Estimación del ahorro hospitalario con el reemplazo de inmunosupresores genéricos en trasplante renal}

\author{
FRANCISCA GONZÁLEZ1 , FERNANDO GONZÁLEZ ${ }^{2}$
}

\section{Cost savings after switching from innovative tacrolimus to a generic version of the drug in transplant recipients}

Background: The use of narrow therapeutic index generic immunosuppressant in solid organ transplantation is controversial. Most experiences switching to these drugs have short term follow-up periods, analyze only pharmacokinetic issues and do not systematically include either complications or cost analyses. Aim: To analyze the costs and benefits of switching our kidney transplant recipients from innovative tacrolimus to a generic version of the drug. Material and Methods: Fifty-seven stable transplant recipients were switched from innovative tacrolimus to a generic version of the drug, maintaining the same dose. They were followed for eight months recording all events during such period. Results: We observed two infectious episodes, five allograft biopsies were performed and two patients had acute rejections. Conclusions: From the payer's perspective, if all the costs associated with the change to generic tacrolimus are considered, savings related to a lower cost of the drug translate in a real financial loss for the public health system. The analysis also showed that frequent switches, even from one generic drug to a cheaper one is an even worse strategy to save money.

(Rev Med Chile 2020; 148: 429-435)

Key words: Costs and Cost Analysis; Drugs, Generic; Immunosuppressive Agents; Transplantation.

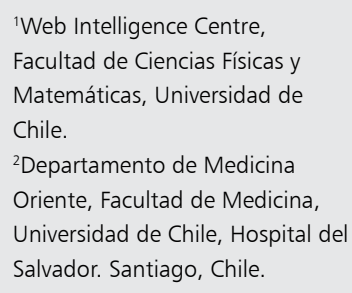

Esta investigación no recibió financiamiento externo. Los autores declaran que no existe conflicto de intereses con respecto a la publicación de este documento.

Recibido el 6 de abril de 2019, aceptado el 16 de abril de 2020 .

Correspondencia a: Fernando González MD, MBA. Facultad de Medicina, Universidad de Chile, Hospital del Salvador. Santiago, Chile. Av. Salvador 467, Providencia, Santiago, Chile. fgonzalf@uc.cl

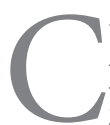
uando un fármaco innovador pierde sus patentes es reemplazado por uno o más genéricos. Estas alternativas más baratas deben cumplir con requisitos técnicos exigentes impuestos por agencias regulatorias, como Food and Drug Administration de los Estados Unidos de Norteamérica (FDA) o European Medicines Agency (EMA), antes de llegar al mercado ${ }^{1,2}$. La eficacia de algunos genéricos ha sido estudiada en experimentos clínicos apropiados y han demostrado ser tan efectivos y seguros como sus contrapartes innovadoras ${ }^{3}$. En el caso de las drogas de estrecho margen terapéutico, como, por ejemplo, los inmunosupresores, tanto médicos como sociedades científicas han sido reacios para aceptar reemplazar las drogas innovadoras por sus genéricos debido a que opinan que pequeñas diferencias farmacocinéticas podrían predisponer a que aparezcan episodios de rechazos agudos o de efectos adversos derivados de toxicidades del producto $^{4,5}$.

Estudios farmacocinéticos han demostrado que, al menos en el corto plazo, los genéricos se comparan favorablemente con los innovadores, lo que ha llevado a concluir que, dadas las diferencias en los precios de compra menores de los genéricos, se justifica su incorporación en los esquemas inmunosupresores reemplazando a los innovadores para obtener ahorros para pacientes, seguros de salud y la sociedad. Expertos más conservadores 
argumentan que en los cálculos de costos totales de tales experiencias no se considera aquellos asociados a los controles médicos más frecuentes, la realización de exámenes, el monitoreo de concentraciones sanguíneas de inmunosupresores ni los costos de diagnosticar y tratar eventuales rechazos agudos o efectos adversos derivados de concentraciones tóxicas de los inmunosupresores ${ }^{4,6}$.

En Chile, la mayoría de los trasplantes renales se realizan en hospitales públicos y son financiados por el seguro público de salud, Fonasa, el que, como organización pública que es, sigue los lineamientos del Ministerio de Salud. Este, instruyó se reemplazara, en todos los trasplantados renales, el tacrolimus innovador por un genérico; dicha experiencia del 2012 fue reportada previamente ${ }^{7}$.

Nuestros objetivos son comunicar los costos involucrados en esa experiencia, explorar si, efectivamente, ese reemplazo fue acertado desde el punto de vista económico y modelar el impacto económico que podría tener el reemplazo periódico de tacrolimus por varios otros genéricos disponibles en el mercado, dado una iniciativa reciente del Ministerio de Salud y la Central Nacional de Abastecimiento (Cenabast) de centralizar las compras de medicamentos de los hospitales para obtener ventajas comerciales derivadas de las compras de volúmenes altos de medicamentos.

\section{Métodos}

La experiencia clínica ${ }^{7}$ consideraba una cohorte de 57 adultos receptores estables de un trasplante renal cambiados desde el tacrolimus innovador (Prograf ${ }^{\circledR}$, Astellas Pharma US, Inc) por un genérico (Cidimus ${ }^{\circledR}$, Sandoz International $\mathrm{GmbH}$, Alemania) en dosis 1:1 y controlados clínicamente por ocho meses; se recopilaron todos los procedimientos médicos y de laboratorio que ocurrieron en ese período.

Los medicamentos concomitantes se mantuvieron estables, pero, podían modificarse si era clínicamente necesario. Todas las drogas eran dispensadas en el hospital, lo mismo que las controles, procedimientos y exámenes diagnósticos.

El sujeto de estudio es el período después del cambio y se lo comparó con los ocho meses previos al reemplazo, por lo tanto, no existió un grupo control conformado por pacientes, ya que el reemplazo de tacrolimus no fue un experimento.
En el seguimiento, observamos: 10 episodios infecciosos, 4 hospitalizados, realizamos 5 biopsias del injerto y dos de ellas mostraron hallazgos compatibles de corresponder a un rechazo agudo. Todos los pacientes se citaron a un control extra para corroborar que el cambio había sido satisfactorio desde el punto de vista clínico y de laboratorio, incluida la concentración sanguínea del tacrolimus.

\section{Información económica}

Utilizando los precios de compra de ambos tacrolimus en el año 2012 y el reembolso de Fonasa para todos los procedimientos clínicos realizados, construimos una función de costo que comparara (A) Ahorros resultantes de las diferencias de precios de adquisición de ambos inmunosupresores con (B) Función de costo de la "inversión" realizada por la institución resultante de la suma de las visitas médicas extra (consulta, exámenes de laboratorio y niveles de tacrolimus) y los costos de los procedimientos diagnósticos y terapéuticos realizados para los eventos descritos más arriba:

A. [Costo del tacrolimus innovador $\mathrm{x}$ Dosis total del período $\mathrm{x}$ Proporción de uso de cada presentación de dosis - Costo del tacrolimus genérico $\mathrm{x}$ Dosis total del período de seguimiento $x$ Proporción de uso de cada presentación de dosis].

B. [Costo de las visitas médicas posterior al reemplazo en 57 pacientes] + [Costo de hospitalizaciones por infecciones $\mathrm{x}$ Probabilidad de ser hospitalizado $(4 / 57)]+[$ Costo de biopsia renal $\mathrm{x}$ Probabilidad de biopsiar (5/57)] + [Costo del tratamiento del rechazo agudo $\mathrm{x}$ Probabilidad de ocurrencia $(2 / 57)]$.

Si costo A > costo B significa que el reemplazo del tacrolimus innovador por el genérico es económicamente conveniente, pero, si costo $\mathrm{A}<$ costo $\mathrm{B}$, implica que tal reemplazo es no conveniente.

\section{Modelamiento del impacto económico de múltiples reemplazos de tacrolimus}

La principal herramienta para evaluar la función del injerto es la creatininemia o la estimación de la velocidad de filtración glomerular (VFGe). Si un trasplantado renal deteriora inexplicadamente su VFGe en $20 \%$ y ello no mejora sustancialmente o empeora en un segundo control, es sometido a una biopsia renal para descartar un rechazo agudo. 
Utilizando las creatininas de los 4 períodos trimestrales previos y posteriores al cambio de tacrolimus, se calculó la variabilidad funcional renal en ambos períodos. Con ello, se estimó, generando números aleatorios mediante el método de Mersenne-Twister, la probabilidad de observar alzas de creatininas que llevaran a practicar una biopsia9. A pesar de contar un buen número de creatininemias en ambos períodos, se lo amplió utilizando bootstraping ${ }^{10}$, que consiste, básicamente, en tomar, en forma reiterada, submuestras aleatorias de la muestras iniciales (198 y 181 datos, respectivamente) para estimar sus estadísticos y, en la medida que esas iteraciones o remuestreos se repitan muchas veces, se logrará obtener una aproximación fidedigna de media y varianza de la variable estudiada (variabilidad funcional renal en nuestro caso). Se realizaron 200 iteraciones adicionales en cada período.

Así, se modeló la probabilidad de realización de biopsias en los períodos previo y posterior al cambio de tacrolimus (Figura 1) y se calculó la función de costo B para cada reemplazo que pudiere hacerse, entendiendo que Cenabast podría hacerlo toda vez que licite inmunosupresores.

Aunque solo disponemos de datos de dos tacrolimus, con los precios de adquisición de todos los tacrolimus disponibles en el mercado $^{11}$ estimaremos, aproximadamente, el ahorro potencial de reemplazos sucesivos a tacrolimus más económicos, ya que licitaciones repetidas permitirían adquirir productos aun más baratos. Como productos de Ascend y Abbot/Recalcine no figuran como aprobados por FDA ni EMA, como sí los otros que se analizarán, se les agregó $10 \%$ de variabilidad adicional para los modelamientos.
Asimismo, utilizando las medias y varianzas de la cantidad de comprimidos de cada presentación del primer genérico, se modeló, mediante bootstrapping ${ }^{10}$, las proporciones de uso de cada presentación para los genéricos disponibles en el mercado.

Este estudio fue aprobado por el Comité de Ética del Servicio de Salud Metropolitano Oriente de Santiago.

\section{Resultados}

De los 57 pacientes adultos (edad promedio 38 años), 41 eran varones (61\%), con un seguimiento posterior al trasplante renal de 49 meses (rango intercuartil 32-65). Tanto concentraciones sanguíneas predosis ( $\mathrm{C} 0)$ de tacrolimus en los dos períodos $(8,0 \pm 2,2$ vs $7,4 \pm 1,6 \mathrm{ng} / \mathrm{mL} ; \mathrm{p}=0,354)$, como dosis diarias $(3,88 \pm 1,98$ versus $4,11 \pm$ $2,05 \mathrm{mg} / \mathrm{d} ; \mathrm{p}=0,308)$, dosis diarias normalizadas por peso corporal $(0,052 \pm 0,023$ versus $0,055 \pm$ $0,033 \mathrm{mg} / \mathrm{Kg} / \mathrm{d} ; \mathrm{p}=0,600)$, como proporciones de uso de las presentaciones de dosis de tacrolimus (cápsulas de $5 \mathrm{mg} / 1 \mathrm{mg} / 0,5 \mathrm{mg}$ : 0,043/0,844/0,113 vs $0,053 / 0,794 / 0,153 ; \mathrm{p}=0,992)$ no difirieron estadísticamente.

Precios de adquisición de los tacrolimus, en dólares (US\$): cápsulas de $5 \mathrm{mg} / 1 \mathrm{mg} / 0,5 \mathrm{mg}$ : Prograf: 11,57/2,15/1,22 vs Cidimus: 8,19/1,64/0,82. Costos, en US\$, asociados a los siguientes procedimientos: Consultas médicas ambulatorias 14, exámenes de laboratorio 20, niveles sanguíneos de tacrolimus 54; visitas médicas hospitalizados 57 , antibióticos 3 , ecografía del injerto 22, biopsia renal 33 , aguja de biopsia 54, procesamiento y

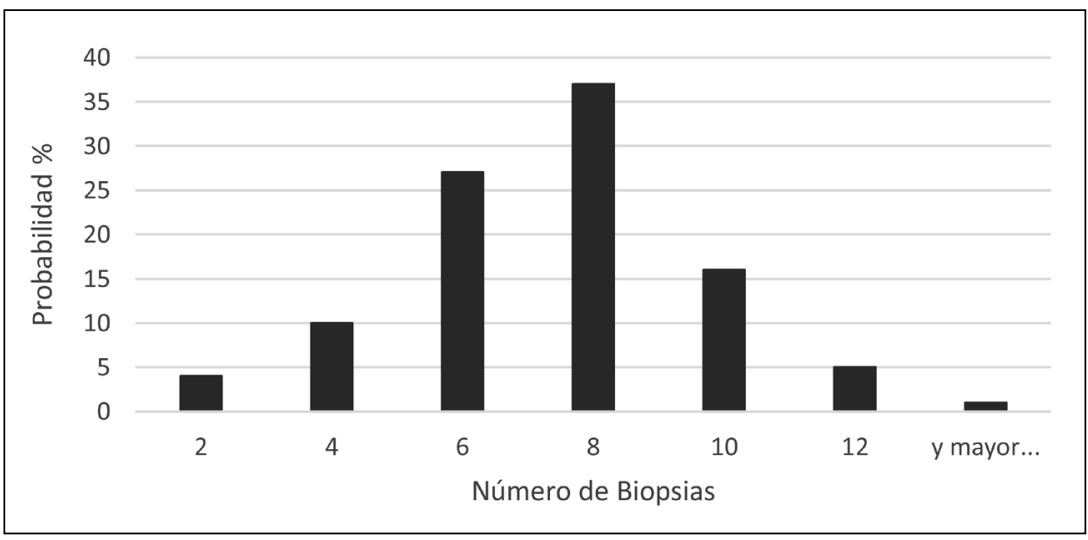

Figura 1. Función de probabilidad de realización de biopsias renales tras el cambio de tacrolimus 
análisis de la biopsia 572 y tratamiento de rechazos agudos 13.093.

Incorporando esos costos en las funciones A y $\mathrm{B}$ resulta en que el reemplazo de Prograf por Cidimus se asoció a una pérdida neta de US\$12.981 (intervalo de confianza 95\% o IC 95\%: Ganancia de US\$ 6.635 hasta pérdida de US\$ 32.598). En otras palabras, después del reemplazo, la probabilidad de perder dinero fue $83,1 \%$ (Tabla 1 ).

La variabilidad de las creatininemias previo y posterior al cambio, definida como [(creatinina actual-creatinina previa)/creatinina previa] fue: $1,01 \pm 0,15$ y $1,02 \pm 0,35$, respectivamente $(\mathrm{p}=0,781)$. Se modeló el número de veces que la creatinina se elevó en $20 \%$ y, al repetir la determinación, ella no mejoró en más de $6 \%$, de tal forma de construir la función de probabilidad de biopsiar el injerto (Figura 1).

Tras repetir el análisis mostrado en la Tabla 1, pero, utilizando los precios de compra de las licitaciones encontradas ${ }^{11}$ (Tabla 2) y las proporciones de uso simuladas (Tabla 3), se obtiene que el reemplazo de Sandoz por Teva se asocia a pérdida financiera neta de US\$ 55.794 (IC 95\%: Pérdidas de US\$ 31.153 hasta US\$ 80.434). El “mejor” de los casos para cambiar Sandoz sería Ascend, que lleva a una pérdida de US\$24.686 (IC 95\%: Pérdidas de US\$ 1.005 hasta US\$ 48.368). La menor

Tabla 1. Matriz de cálculo de costos en US\$ del reemplazo del tacrolimus innovador al genérico

\begin{tabular}{|c|c|c|c|c|c|c|c|c|c|c|}
\hline & $\begin{array}{c}\text { Dosis } \\
\text { promedio } \\
\text { (mg) }\end{array}$ & DS & $\begin{array}{l}\text { Pacien- } \\
\text { tes }\end{array}$ & $\begin{array}{l}\text { Meses } \\
\text { de uso }\end{array}$ & $\begin{array}{l}\text { Dosis } \\
\text { total }\end{array}$ & $\begin{array}{l}\text { Margen } \\
\text { inferior }\end{array}$ & $\begin{array}{l}\text { Margen } \\
\text { superior }\end{array}$ & $\begin{array}{c}\text { Costo } \\
\text { del } \\
\text { período }\end{array}$ & $\begin{array}{l}\text { Margen } \\
\text { inferior }\end{array}$ & $\begin{array}{l}\text { Margen } \\
\text { superior }\end{array}$ \\
\hline Tacrolimus innovador & 3,88 & 1,98 & 57 & 8 & 53.078 & 51.235 & 54.922 & 168.724 & 162.863 & 174.585 \\
\hline Tacrolimus genérico & 4,11 & 2,05 & 57 & 8 & 56.225 & 54.203 & 58.247 & 145.764 & 140.522 & 151.007 \\
\hline \multirow{2}{*}{\multicolumn{11}{|c|}{ Reemplazo }} \\
\hline & & & & & & & & & & \\
\hline Visitas extras & & 57 & & & & & & 820 & 820 & 820 \\
\hline Exámenes de laboratorio & & 57 & & & & & & 1.156 & 1.156 & 1.156 \\
\hline $\begin{array}{l}\text { Monitoreo niveles sanguíneos } \\
\text { tacrolimus }\end{array}$ & & 57 & $\%$ & $\begin{array}{l}\text { Error } \\
\text { estándar }\end{array}$ & & & & 3.083 & 3.083 & 3.083 \\
\hline Hospitalización por infección & & 4 & 7,02 & 3,38 & & & & 717 & 371 & 1.063 \\
\hline $\begin{array}{l}\text { Hospitalización para realización } \\
\text { de biopsia del injerto }\end{array}$ & & 5 & 8,77 & 3,75 & & & & 3.973 & 2.276 & 5.671 \\
\hline \multirow[t]{3}{*}{ Episodio de rechazo agudo } & & 2 & 3,51 & 2,44 & & & & 26.191 & 7.999 & 44.383 \\
\hline & & & & & & & $\begin{array}{l}\text { "Inversión" } \\
\text { (B) }\end{array}$ & 35.941 & 15.706 & 56.176 \\
\hline & & & & & \multicolumn{3}{|c|}{$\begin{array}{l}\text { Resultado económico } \\
\text { del reemplazo (A-B) }\end{array}$} & $(12.981)$ & 6.635 & (32.598) \\
\hline
\end{tabular}

Tabla 2. Precios de cápsulas de tacrolimus ofertados en mercadopublico.cl en US\$11

\begin{tabular}{|cccccc|}
\hline & Astellas & Sandoz & Teva & Ascend & Recalcine/Abbott \\
\hline $0,5 \mathrm{mg}$ & 0,695 & 0,494 & 0,525 & 0,255 & 0,794 \\
$1,0 \mathrm{mg}$ & 1,374 & 0,787 & 0,695 & 0,324 & 0,479 \\
\hline $5,0 \mathrm{mg}$ & 6,948 & 4,014 & 4,941 & 3,088 & 3,706 \\
\hline
\end{tabular}


Tabla 3. Proporción de uso de cápsulas de cada presentación*

\begin{tabular}{|cccccc|}
\hline Proporción de uso & Prograf & Sandoz & Teva & Ascend & Recalcine/Abbott \\
\hline $0,5 \mathrm{mg}$ & 0,043 & 0,053 & 0,015 & 0,021 & 0,051 \\
$1,0 \mathrm{mg}$ & 0,844 & 0,794 & 0,832 & 0,81 & 0,746 \\
\hline $5,0 \mathrm{mg}$ & 0,113 & 0,153 & 0,153 & 0,169 & 0,203 \\
\hline
\end{tabular}

*Recalcine/Abbott no posee registros de todas las presentaciones en el ISP.

Tabla 4. Matriz de cálculo de costos en US\$ del reemplazo de un tacrolimus actual a otro adquirido según licitaciones repetidas ${ }^{8}$

\begin{tabular}{|llllllll|}
\hline Inicial & Cambio & $\begin{array}{c}\text { Ahorro } \\
\text { compra } \\
\text { US\$ }\end{array}$ & \% Ahorro & $\begin{array}{c}\text { Ahorro } \\
\text { REAL US\$ }\end{array}$ & $\begin{array}{c}\text { Margen } \\
\text { inferior } \\
\text { US\$ }\end{array}$ & $\begin{array}{c}\text { Margen } \\
\text { superior } \\
\text { US\$ }\end{array}$ & $\begin{array}{c}\text { \% Ahorro } \\
\text { REAL }\end{array}$ \\
\hline Prograf & Sandoz & $33.882,54$ & $32,28 \%$ & $-2.020,90$ & $17.103,28$ & $-21.145,08$ & $-1,93 \%$ \\
\hline Sandoz & Teva & $-4.426,91$ & $-6,23 \%$ & $-55.793,76$ & $-31.153,31$ & $-80.434,22$ & $-78,48 \%$ \\
& Recalcine & $6.469,52$ & $9,10 \%$ & $-44.897,32$ & $-20.416,35$ & $-69.378,30$ & $-63,15 \%$ \\
& Ascend & $26.680,63$ & $37,53 \%$ & $-24.686,22$ & $-1.004,82$ & $-48.367,61$ & $-34,72 \%$ \\
\hline Teva & Recalcine & $10.896,44$ & $14,43 \%$ & $-40.470,41$ & $-16.148,65$ & $-64.792,17$ & $-53,59 \%$ \\
& Ascend & $31.107,55$ & $41,19 \%$ & $-20.259,30$ & $3.262,88$ & $-43.781,49$ & $-26,83 \%$ \\
\hline \multirow{2}{*}{ Recalcine } & Ascend & $20.211,11$ & $31,28 \%$ & $-31.155,74$ & $-7.474,08$ & $-54.837,40$ & $-48,21 \%$ \\
\hline
\end{tabular}

pérdida sería desde Teva a Ascend, de US\$ 20.259 (IC 95\%: Ganancia de US\$ 3.263 hasta pérdida de US\$ 43.781). En otras palabras, tras reemplazos de tacrolimus, la probabilidad de perder dinero se estima en 92,6\% (Rango 55-100\%) (Tabla 4).

La diferente cuantía de los resultados de las Tablas 1 y 4, (reemplazo de Astellas por Sandoz) se explica por las caídas de precios entre los años 2012 y 2018, donde todas las presentaciones de Sandoz bajaron $50 \%$ o más, mientras que Astellas las redujo en menor proporción, lo que hace el ahorro del cambio mayor desde el punto de vista del comprador de medicamentos.

\section{Discusión}

Aunque los precios de los genéricos son menores, el costo total del reemplazo en trasplantados renales podría no ser tan conveniente como podría parecer a primera vista si se agregan todos los costos secundarios: controles médicos extra, monitoreo de concentraciones sanguíneas de fármacos y diagnóstico y tratamiento de compli- caciones eventuales, por ejemplo, disminución de la VFG debido a toxicidad del fármaco, infección o episodios de rechazo agudo.

Esto es progresivamente más relevante mientras más genéricos ingresan al mercado, presionando los precios a la baja y transformando las licitaciones en verdaderas batallas para lograr mejores cuotas de mercado. De hecho, si las diferencias en los precios disminuyen, tal como es habitual de observar ${ }^{12}$, los ahorros reales de reemplazar innovadores por genéricos o, más aun, entre genéricos, desaparecen por completo y los costos secundarios e inadvertidos, al mantenerse constantes, tenderán a arruinar a quienes administran los seguros de salud.

En los estudios clínicos existen al menos dos brazos experimentales, uno con la intervención innovadora y otro que mantiene el estándar (grupo control). En este estudio observacional, lo que se comparan son los períodos antes y después del reemplazo y no los pacientes en sí mismos. En estos casos, el uso de herramientas de simulación permite el hacerse una idea de los posibles cursos de la intervención. Tanto la generación de 
números aleatorios ${ }^{9}$ como el bootstrapping ${ }^{10}$ dan la posibilidad de explorar el devenir de acontecimientos tras la repetición, al menos teórica, de lo que podría ocurrir al reemplazar repetidamente el tacrolimus en el esquema inmunosupresor.

Es sabido que la tasa de episodios de rechazo agudo es más alta cerca del trasplante y que disminuye hasta aproximarse asintóticamente a un valor bajo ${ }^{13-15}$. Desde la perspectiva matemática, esto significa que cuando la tasa se acerca a hacerse constante, la probabilidad de observar un nuevo episodio de rechazo agudo es despreciable. Cada inmunosupresor tiene su propia curva $y$, cuando se reemplaza un agente por otro, la probabilidad de ocurrencia de un rechazo agudo vuelve a aumentar, tal como si la "historia regresara al pasado".

La aparición de dos nuevos episodios de rechazo agudo tardío (3 años postrasplante) es una señal de advertencia de que algo malo podría haber ocurrido.

Otro factor potencialmente aumentador del costo total del cambio es el comportamiento de los médicos, quienes, obedeciendo guías clínicas ${ }^{4,5}$, podrían controlar más frecuentemente a los pacientes, sesgarse y realizar más biopsias o ser más suspicaces para diagnosticar y tratar episodios de rechazo con el fin de que no disminuir la calidad de la atención brindada. Esta posibilidad podría ser aun más trascendental si las licitaciones reiteradas de fármacos son percibidas, por ellos mismos, como injustas o incapaces de entregar un tratamiento inmunosupresor adecuado a los trasplantados. Los principios éticos se centran en las personas individuales, la seguridad del paciente en este caso, en contraposición con los administradores de las instituciones que entregan atenciones de salud o quienes manejan los seguros de salud, cuyo objetivo es ahorrar dinero para ser más eficientes.

Es interesante ver que gran parte de nuestros hallazgos en el análisis económico y en las simulaciones de cambios reiterados; tras licitaciones repetidas ${ }^{8}$, obedecen a la mayor variabilidad en la función renal que aparece tras el reemplazo de productos, lo cual, aunque no sea estadísticamente diferente respecto del período previo al cambio, sí obliga a la realización de más biopsias del injerto $y$, consecuentemente, al hallazgo de más rechazos agudos que, dado su enorme costo unitario (US\$ 13.093) amplifican las cifras al estimar costos y sus consecuentes resultados económicos.
En Chile, la complicación más costosa después del trasplante es el rechazo agudo, que contempla un promedio ponderado de los costos de todos los rechazos, incluidos los rechazos celulares y los mediados por anticuerpos, que necesitan ser tratados, inclusive, con inmunoglobulina intravenosa, plasmaféresis y anticuerpos monoclonales. Esto explica por qué el rechazo agudo es el principal impulsor de costos en nuestro análisis y por qué, desde una perspectiva netamente económica, no importa si esta complicación podría haber ocurrido de todos modos, incluso sin haber reemplazado el tacrolimus, porque toda la historia previa de cada paciente antes del cambio es, en realidad, costo hundido, es decir, recursos que ya se destinaron y no pueden ser recuperados. Más aun, en nuestras simulaciones en el período basal, no encontramos deterioros funcionales que motivaran el biopsiar, como sí ocurrió frecuentemente en el período tras los reemplazos (Figura 1).

Concluimos que cambiar inmunosupresores, ya sea desde el innovador a un genérico o, más aun, entre genéricos, es muy poco probable que terminen siendo económicamente convenientes. Aunque los genéricos sean más baratos que sus homólogos innovadores y que nuevos genéricos lo sean aun más, la suma de los costos asociados a dichos costos pueden transformar un ahorro hipotético en una desastrosa pérdida financiera para el sistema de seguro de salud.

Es necesario el realizar simulaciones y análisis económicos cuidadosos antes de implementar políticas públicas, especialmente, si las consecuencias de esas políticas puedan ser trascendentales. En nuestro análisis solo hemos considerado el trasplante renal que, en el peor de los casos, si es que este falla, existe la posibilidad de diálisis crónica, pero si el trasplante es de corazón, hígado o pulmón, la consecuencia puede ser una muerte y, lo que es peor, que pudo haber sido evitada.

\section{Referencias}

1. Generic Drugs: Questions and Answers. http://www. fda.gov/Drugs/ResourcesForYou/Consumers/QuestionsAnswers/ucm100100.htm. Access July 8, 2018

2. Generic/ hybrid applications: questions and answers. http://www.ema.europa.eu/ema/index.jsp?curl=pages/ regulation/general/general_content_000179.jsp\&mi-

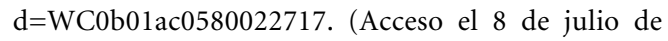
2018). 
3. Kesselheim A, Misono A, J Lee, Stedman M, Brookhart $\mathrm{M}$, Choudhry N, et al. Clinical Equivalence of Generic and Brand-Name Drugs Used in Cardiovascular Disease: A Systematic Review and Meta-analysis. JAMA. 2008 Dec 3; 300: 2514-26. doi: 10.1001/jama.2008.758.

4. van Gelder T. European Society for Organ Transplantation Advisory Committee Recommendations on Generic Substitution of Immunosuppressive Drugs. Transpl Int 2011; 24: 1135-41. doi: 10.1111/j.14322277.2011.01378.x

5. Alloway R, Isaacs R, Lake K, Hoyer P, First R, Helderm $\mathrm{H}$, et al. Report of the American Society of Transplantation conference on immunosuppressive drugs and the use of generic immunosuppressants. Am J Transplant 2003; 3: 1211-15. doi.org/10.1046/j.16006143.2003.00212.x

6. van Gelder T, Gabardi S. Methods, strengths, weaknesses, and limitations of bioequivalence tests with special regard to immunosuppressive drugs. Transpl Int 2013; 26: 771-7. doi.org/10.1111/tri.12074.

7. González F, López R, Arriagada E, Carrasco R, Gallardo N, Lorca E. Switching Stable Kidney Transplant Recipients to a Generic Tacrolimus Is Feasible and Safe, but It Must Be Monitored. J Transplant 2017, Article ID 5646858. https://doi.org/10.1155/2017/5646858.

8. Cenabast. El proyecto Catálogo de Compras fue una de las novedades exhibidas en la Expo Mercado Público 2018. https://www.cenabast.cl/el-proyecto-catalogo-de-compras-fue-una-de-las-novedades-exhibidas-en-la-expo-mercado-publico-2018/ (Acceso el 26 de enero de 2019).

9. Matsumoto M, Nishimura T. Mersenne twister: a 623-dimensionally equidistributed uniform pseudo-random number generator. ACM Transactions on Modeling and Computer Simulation 1998; 8 (1): 3-30.

10. Efron B. Bootstrap methods: Another look at the jackknife. The Annals of Statistics 1979; 7 (1): 1-26.

11. Búsqueda de licitaciones cerradas de tacrolimus 2018. http://www.mercadopublico.cl/Portal/Modules/Site/ Busquedas/ResultadoBusqueda.aspx?qs=1\&IdEmpresa= (Acceso el 26 de enero de 2019).

12. Richard G. Frank. The Ongoing Regulation of Generic Drugs. N Engl J Med 2007; 357:1993-6. DOI: 10.1056/ NEJMp078193.

13. Keown P, Niese D on behalf of the International Sandimmun Neoral Study Group. Cyclosporine microemulsion increases drug exposure and reduces acute rejection without incremental toxicity in de novo renal transplantation. Kidney Int 1998; 54: 938-44. doi.org/10.1046/j.1523-1755.1998.00042.x

14. Flechner S, Glyda M, Cockfield S, Grinyó J, Legendre Ch, Russ G, et al. The ORION Study: Comparison of Two Sirolimus-Based Regimens versus Tacrolimus and Mycophenolate Mofetil in Renal Allograft Recipients. Am J Transplant 2011; 11: 1633-44. doi: 10.1111/j.16006143.2011.03573.x

15. H Silva Jr, Yang H, Abouljoud M, Kuo P, Wisemandle $\mathrm{K}$, Bhattacharya $\mathrm{P}$, et al. for the Tacrolimus Extended-Release De Novo Kidney Study Group. One-Year Results with Extended-Release Tacrolimus/ MMF, Tacrolimus/MMF and Cyclosporine/MMF in De Novo Kidney Transplant Recipients. Am J Transplant 2007; 11: 595-608. doi.org/10.1111/j.16006143.2007.01661.x 